IoAnnis K. Argyros (Lawton, OK)

SAÏD Hilout (Béni-Mellal)

\title{
NEWTON'S METHODS FOR VARIATIONAL INCLUSIONS UNDER CONDITIONED FRÉCHET DERIVATIVE
}

Abstract. Estimates of the radius of convergence of Newton's methods for variational inclusions in Banach spaces are investigated under a weak Lipschitz condition on the first Fréchet derivative. We establish the linear convergence of Newton's and of a variant of Newton methods using the concepts of pseudo-Lipschitz set-valued map and $\omega$-conditioned Fréchet derivative or the center-Lipschitz condition introduced by the first author.

1. Introduction. This paper is concerned with the problem of approximating a solution of the variational inclusion

$$
0 \in f(x)+G(x)
$$

where $f: X \rightarrow Y$ is a continuous function and $G: X \rightarrow 2^{Y}$ is a set-valued map with closed graph; $X$ and $Y$ are Banach spaces. Some problems related to mathematical programming, complementarity problems, optimal control and to other fields can be represented in the form (1.1) (see for example $[21,22])$.

For approximating locally the unique solution $x^{*}$ of (1.1), we consider the Newton method based on the following partial linearization:

$$
\left\{\begin{array}{l}
x_{0} \text { is given as starting point, } \\
0 \in f\left(x_{k}\right)+\nabla f\left(x_{k}\right)\left(x_{k+1}-x_{k}\right)+G\left(x_{k+1}\right),
\end{array}\right.
$$

where $\nabla f(x)$ denotes the Fréchet derivative of $f$ at $x$. Dontchev [8] proved that Newton's method (1.2) is quadratically convergent to $x^{*}$ under the pseudo-Lipschitzianity of $(f+G)^{-1}$ and the Lipschitz continuity of $\nabla f$ in a

2000 Mathematics Subject Classification: 47H04, 65K10, $49 \mathrm{~J} 53$.

Key words and phrases: Newton's method, set-valued map, $\omega$-condition, variational inclusions, Aubin continuity. 
neighborhood $V$ of $x^{*}$ with constant $L$ :

$$
\|\nabla f(x)-\nabla f(y)\| \leq L\|x-y\|, \quad x, y \in V .
$$

Piétrus [20] obtained superlinear convergence whenever the Fréchet derivative of $f$ satisfies a Hölder condition

$$
\|\nabla f(x)-\nabla f(y)\| \leq L\|x-y\|^{p}, \quad x, y \in V, p \in[0,1] .
$$

A similar condition to (1.4) on the first order divided difference is used in [13]-[16] to study the local convergence for the secant and Steffensentype methods. Some convergence analysis of (1.2) is presented in [2] using a condition on the $m$ th $(m \geq 2)$ Fréchet derivative $\nabla^{(m)} f$ :

$$
\left\|\nabla^{(m)} f(x)-\nabla^{(m)} f\left(x_{0}\right)\right\| \leq L\left\|x-x_{0}\right\|
$$

for $x$ in some neighborhood of $x_{0}$.

For the case of nonlinear equations $(G \equiv 0$ in (1.1)), algorithm (1.2) is reduced to Newton's method for solving $f(x)=0$ and has been widely studied these last years (see for example $[1,3,4,11]$ and the references given there).

In [17], the authors present the following variant of Newton's method:

$$
\left\{\begin{array}{l}
y_{0} \text { is given as starting point, } \\
0 \in f\left(y_{k}\right)+h \nabla f\left(y_{k}\right)\left(y_{k+1}-y_{k}\right)+G\left(y_{k+1}\right),
\end{array}\right.
$$

where $h$ is a constant $(h \neq 1)$. Local linear convergence is investigated in [17] under Lipschitz condition (1.3).

In this paper, we use different conditions from the previous ones to study the convergence of Newton's method (1.2) and of the variant (1.6). We relax the usual Lipschitz and Hölder conditions. So, the main conditions required are

$$
\begin{aligned}
\|\nabla f(x)-\nabla f(y)\| & \leq \omega(\|x-y\|) & & \text { for } x, y \text { in } V, \\
\left\|\nabla f(x)-\nabla f\left(x^{*}\right)\right\| & \leq \mu\left(\left\|x-x^{*}\right\|\right) & & \text { for } x \text { in } V,
\end{aligned}
$$

where $\omega, \mu: \mathbb{R}_{+} \rightarrow \mathbb{R}_{+}$are continuous nondecreasing functions. When (1.7) is satisfied, we say that $\nabla f$ is $\omega$-conditioned. Condition (1.8) is called the center-Lipschitz condition on the operator $\nabla f$. Conditions (1.7) and (1.8) are used in $[1,4,5,12]$ to solve nonlinear equations.

Our main tool used for obtaining linear convergence is the Aubin continuity of $\left(f\left(x^{*}\right)+\nabla f\left(x^{*}\right)\left(\cdot-x^{*}\right)+G(\cdot)\right)^{-1}$ at $\left(0, x^{*}\right)$. Before defining this concept, let us give some standard notations. We denote by $\mathbb{B}_{r}(x)$ the closed ball centered at $x$ with radius $r$, and by $\|\cdot\|$ the norms of $X$ and $Y$; the distance from a point $x \in X$ to a subset $A \subset X$ is defined as $\operatorname{dist}(x, A)=\inf _{a \in A}\|x-a\|$. Let $\Lambda: X \rightarrow 2^{Y}$ be a set-valued map. We write $\operatorname{gph} \Lambda=\{(x, y) \in X \times Y: y \in \Lambda(x)\}$ and $\Lambda^{-1}(y)=\{x \in X: y \in \Lambda(x)\}$. A set-valued map $\Gamma: X \rightarrow 2^{Y}$ is said to be M-pseudo-Lipschitz around 
$\left(x_{0}, y_{0}\right) \in \operatorname{gph} \Gamma(M>0)$ (or Aubin continuous at $\left.\left(x_{0}, y_{0}\right)\right)$ if there exist constants $a$ and $b$ such that

$$
e\left(\Gamma\left(x_{1}\right) \cap \mathbb{B}_{a}\left(y_{0}\right), \Gamma\left(x_{2}\right)\right) \leq M\left\|x_{1}-x_{2}\right\|, \quad \forall x_{1}, x_{2} \in \mathbb{B}_{b}\left(x_{0}\right),
$$

where the excess from the set $A$ to the set $C$ is defined by $e(C, A)=$ $\sup _{x \in C} \operatorname{dist}(x, A)$.

The pseudo-Lipschitz property has been introduced by Aubin (see [6]). A basic characterization of this property of the inverse of a set-valued map is given by the Graves theorem (see [9]). Other characterizations of Aubin continuity have been obtained by Rockafellar [23] using the Lipschitz continuity of the distance function $(x, y) \mapsto \operatorname{dist}(y, \Gamma(x))$ around $\left(x_{0}, y_{0}\right)$, and by Mordukhovich $[18,24]$ via the concept of coderivative of multifunctions $\mathcal{D}^{*} \Gamma(x / y)$, where

$$
v \in \mathcal{D}^{*} \Gamma(x / y)(u) \Leftrightarrow(v,-u) \in N_{\mathrm{gph}}(x, y) .
$$

The Mordukhovich criterion says that $\Gamma$ with a closed graph is pseudoLipschitz around $\left(x_{0}, y_{0}\right)$ if and only if

$$
\left\|\mathcal{D}^{*} \Gamma\left(x_{0} / y_{0}\right)\right\|^{+}=\sup _{u \in \mathbb{B}_{1}(0)} \sup _{v \in \mathcal{D}^{*} \Gamma\left(x_{0} / y_{0}\right)(u)}\|v\|<\infty .
$$

In fact, the Mordukhovich criterion plays a fundamental role in variational analysis and its applications. We refer the reader to $[6,7,9,10,18,19,23,24]$ and the references given there for more details and applications of Aubin continuity.

2. Preliminaries and assumptions. As the main tool of our analysis we will use the following lemmas. The first is the fixed point theorem for set-valued map proved by Dontchev and Hager [9]. This theorem is a generalization of the Picard fixed point theorem restricted to single-valued mappings. The second lemma gives an estimate using condition (1.7).

Lemma 2.1 (see [9]). Let $\phi$ be a set-valued map from $X$ into the closed subsets of $X$, let $\eta_{0} \in X$, and let $r$ and $\lambda$ be such that $0 \leq \lambda<1$ and

(a) $\operatorname{dist}\left(\eta_{0}, \phi\left(\eta_{0}\right)\right) \leq r(1-\lambda)$.

(b) $e\left(\phi\left(x_{1}\right) \cap \mathbb{B}_{r}\left(\eta_{0}\right), \phi\left(x_{2}\right)\right) \leq \lambda\left\|x_{1}-x_{2}\right\|$ for all $x_{1}, x_{2} \in \mathbb{B}_{r}\left(\eta_{0}\right)$.

Then $\phi$ has a fixed point in $\mathbb{B}_{r}\left(\eta_{0}\right)$, that is, there exists $x \in \mathbb{B}_{r}\left(\eta_{0}\right)$ such that $x \in \phi(x)$. If $\phi$ is single-valued, then $x$ is the unique fixed point of $\phi$ in $\mathbb{B}_{r}\left(\eta_{0}\right)$.

Lemma 2.2. Suppose that the assumption (1.7) is satisfied on a convex neighborhood $V$. Then for all $x$ and $y$ in $V$,

$$
\|f(x)-f(y)-\nabla f(y)(x-y)\| \leq \mu(\|x-y\|)\|x-y\| .
$$


In particular, if the assumption (1.8) is satisfied then for all $x$ in $V$,

$$
\left\|f(x)-f\left(x^{*}\right)-\nabla f\left(x^{*}\right)\left(x-x^{*}\right)\right\| \leq \mu\left(\left\|x-x^{*}\right\|\right)\left\|x-x^{*}\right\| .
$$

Proof. For all $x$ and $y$ in $V$ we can write

$$
f(x)-f(y)-\nabla f(y)(x-y)=\left(\int_{0}^{1} \nabla f(x+t(y-x)) d t-\int_{0}^{1} \nabla f(y) d t\right)(x-y) .
$$

Hence

$$
\|f(x)-f(y)-\nabla f(y)(x-y)\| \leq\|x-y\| \int_{0}^{1}\|\nabla f(x+t(y-x)) d t-\nabla f(y)\| d t .
$$

Before stating the main result of this study, we need to introduce some notations. First, for $k \in \mathbb{N}$ and $\left(x_{k}\right)$ defined in (1.2), define the set-valued mappings $Q: X \rightarrow 2^{Y}$ and $\psi_{k}: X \rightarrow 2^{X}$ by

$$
Q(\cdot):=f\left(x^{*}\right)+\nabla f\left(x^{*}\right)\left(\cdot-x^{*}\right)+G(\cdot), \quad \psi_{k}(\cdot):=Q^{-1}\left(Z_{k}(\cdot)\right),
$$

where $Z_{k}: X \rightarrow Y$ is defined by

$$
Z_{k}(x):=f\left(x^{*}\right)+\nabla f\left(x^{*}\right)\left(x-x^{*}\right)-f\left(x_{k}\right)-\nabla f\left(x_{k}\right)\left(x-x_{k}\right) .
$$

Note that $x_{1}$ is a fixed point of $\psi_{0}$ if and only if $0 \in f\left(x_{0}\right)+\nabla f\left(x_{0}\right)\left(x_{1}-\right.$ $\left.x_{0}\right)+G\left(x_{1}\right)$.

We will make the following assumptions in an open convex neighborhood $V$ of $x^{*}$ :

$(\mathcal{H} 1)$ The condition (1.7) is satisfied on $V$.

$(\mathcal{H} 2)$ The set-valued map $\left(f\left(x^{*}\right)+\nabla f\left(x^{*}\right)\left(\cdot-x^{*}\right)+G(\cdot)\right)^{-1}$ is $M$-pseudoLipschitz around $\left(0, x^{*}\right)$ with constants $a$ and $b$ (see the definition of Aubin continuity) and $M \omega(a)<1 / 2$.

3. Convergence analysis. In this section we are concerned with the existence of sequences $\left(x_{n}\right)$ satisfying (1.2) and the $q$-linear convergence of $\left(x_{n}\right)$ to the solution $x^{*}$ of (1.1) under the previous assumptions. The main result of this study is as follows.

TheOREM 3.1. Let $x^{*}$ be a solution of (1.1). Suppose that assumptions $(\mathcal{H} 1)-(\mathcal{H} 2)$ are satisfied. For every constant $C$ such that

$$
\frac{M \omega(a)}{1-M \omega(a)}<C<1
$$

one can find $\delta>0$ such that for every starting point $x_{0} \neq x^{*}$ in $\mathbb{B}_{\delta}\left(x^{*}\right)$, there exists a sequence $\left(x_{k}\right)$ satisfying $(1.2)$ which is q-linearly convergent to $x^{*}$, i.e.,

$$
\left\|x_{k+1}-x^{*}\right\| \leq C\left\|x_{k}-x^{*}\right\| .
$$


The proof of Theorem 3.1 is by induction on $k$. We prove the existence of a starting point $x_{1}$ for $x_{0}$ in $V$.

Proposition 3.2. Under the assumptions of Theorem 3.1, one can find $\delta>0$ such that for every starting point $x_{0} \neq x^{*}$ in $\mathbb{B}_{\delta}\left(x^{*}\right)$, the set-valued map $\psi_{0}$ has a fixed point $x_{1}$ in $\mathbb{B}_{\delta}\left(x^{*}\right)$ satisfying

$$
\left\|x_{1}-x^{*}\right\| \leq C\left\|x_{0}-x^{*}\right\|,
$$

where $C$ is as in Theorem 3.1.

Proof. By hypothesis $(\mathcal{H} 2)$ we have

$$
e\left(Q^{-1}\left(y^{\prime}\right) \cap \mathbb{B}_{a}\left(x^{*}\right), Q^{-1}\left(y^{\prime \prime}\right)\right) \leq M\left\|y^{\prime}-y^{\prime \prime}\right\|, \quad \forall y^{\prime}, y^{\prime \prime} \in \mathbb{B}_{b}(0) .
$$

Fix $\delta>0$ such that

$$
\delta<\min \left\{a, \frac{b}{2 \omega(a)}\right\} .
$$

The main idea of the proof is to show that both assertions (a) and (b) of Lemma 2.1 hold, where $\eta_{0}:=x^{*}, \phi=\psi_{0}$ defined in (2.1), and where $r$ and $\lambda$ are numbers to be set. According to the definition of the excess $e$, we have

$$
\operatorname{dist}\left(x^{*}, \psi_{0}\left(x^{*}\right)\right) \leq e\left(Q^{-1}(0) \cap \mathbb{B}_{\delta}\left(x^{*}\right), \psi_{0}\left(x^{*}\right)\right) .
$$

For all $x_{0}$ in $\mathbb{B}_{\delta}\left(x^{*}\right)$, by assumptions $(\mathcal{H} 1),(3.4)$ and Lemma 2.2 we have

$$
\begin{aligned}
\left\|Z_{0}\left(x^{*}\right)\right\| & =\left\|f\left(x^{*}\right)-f\left(x_{0}\right)-\nabla f\left(x_{0}\right)\left(x^{*}-x_{0}\right)\right\| \\
& \leq \omega\left(\left\|x_{0}-x^{*}\right\|\right)\left\|x_{0}-x^{*}\right\| \leq \omega(a)\left\|x_{0}-x^{*}\right\| .
\end{aligned}
$$

Then (3.4) yields $Z_{0}\left(x^{*}\right) \in \mathbb{B}_{b}(0)$. Consequently,

$$
\begin{aligned}
e\left(Q^{-1}(0) \cap \mathbb{B}_{\delta}\left(x^{*}\right), \psi_{0}\left(x^{*}\right)\right) & =e\left(Q^{-1}(0) \cap \mathbb{B}_{\delta}\left(x^{*}\right), Q^{-1}\left[Z_{0}\left(x^{*}\right)\right]\right) \\
& \leq M \omega(a)\left\|x_{0}-x^{*}\right\| .
\end{aligned}
$$

By inequality (3.5), we get

$$
\operatorname{dist}\left(x^{*}, \psi_{0}\left(x^{*}\right)\right) \leq M \omega(a)\left\|x_{0}-x^{*}\right\| .
$$

Since $C(1-M \omega(a))>M \omega(a)$, there exists $\lambda \in[M \omega(a), 1 / 2[$ such that $C(1-\lambda) \geq M \omega(a)$ and

$$
\operatorname{dist}\left(x^{*}, \psi_{0}\left(x^{*}\right)\right) \leq C(1-\lambda)\left\|x_{0}-x^{*}\right\| .
$$

By choosing $r:=r_{0}=C\left\|x_{0}-x^{*}\right\|$ we deduce from (3.9) that assertion (a) in Lemma 2.1 is satisfied.

By (3.4) we have $r_{0} \leq \delta \leq a$. Moreover, for $x \in \mathbb{B}_{\delta}\left(x^{*}\right)$, by $(\mathcal{H} 1),(3.4)$ and Lemma 2.2 we have 


$$
\begin{aligned}
\left\|Z_{0}(x)\right\|= & \left\|f\left(x^{*}\right)+\nabla f\left(x^{*}\right)\left(x-x^{*}\right)-f\left(x_{0}\right)-\nabla f\left(x_{0}\right)\left(x-x_{0}\right)\right\| \\
\leq & \left\|f\left(x^{*}\right)-f\left(x_{0}\right)-\nabla f\left(x_{0}\right)\left(x^{*}-x_{0}\right)\right\| \\
& +\left\|\left(\nabla f\left(x^{*}\right)-\nabla f\left(x_{0}\right)\right)\left(x-x^{*}\right)\right\| \\
\leq & \omega\left(\left\|x_{0}-x^{*}\right\|\right)\left\|x_{0}-x^{*}\right\|+\left\|\nabla f\left(x^{*}\right)-\nabla f\left(x_{0}\right)\right\|\left\|x-x^{*}\right\| \\
\leq & \omega(a)\left\|x_{0}-x^{*}\right\|+\omega\left(\left\|x_{0}-x^{*}\right\|\right)\left\|x-x^{*}\right\| \leq 2 \omega(a) \delta .
\end{aligned}
$$

Thus by (3.4) we deduce that $Z_{0}(x) \in \mathbb{B}_{b}(0)$ for all $x \in \mathbb{B}_{\delta}\left(x^{*}\right)$. It follows that for all $x^{\prime}, x^{\prime \prime} \in \mathbb{B}_{r_{0}}\left(x^{*}\right)$,

$$
e\left(\psi_{0}\left(x^{\prime}\right) \cap \mathbb{B}_{r_{0}}\left(x^{*}\right), \psi_{0}\left(x^{\prime \prime}\right)\right) \leq e\left(\psi_{0}\left(x^{\prime}\right) \cap \mathbb{B}_{\delta}\left(x^{*}\right), \psi_{0}\left(x^{\prime \prime}\right)\right),
$$

which yields, by (3.3),

$$
\begin{aligned}
e\left(\psi_{0}\left(x^{\prime}\right) \cap \mathbb{B}_{r_{0}}\left(x^{*}\right), \psi_{0}\left(x^{\prime \prime}\right)\right) & \leq M\left\|Z_{0}\left(x^{\prime}\right)-Z_{0}\left(x^{\prime \prime}\right)\right\| \\
& =M\left\|\left(\nabla f\left(x^{*}\right)-\nabla f\left(x_{0}\right)\right)\left(x^{\prime \prime}-x^{\prime}\right)\right\| \\
& \leq M\left\|\nabla f\left(x^{*}\right)-\nabla f\left(x_{0}\right)\right\|\left\|x^{\prime \prime}-x^{\prime}\right\| \\
& \leq M \omega(a)\left\|x^{\prime \prime}-x^{\prime}\right\| .
\end{aligned}
$$

The inequality $\lambda \geq M \omega(a)$ implies

$$
e\left(\psi_{0}\left(x^{\prime}\right) \cap \mathbb{B}_{r_{0}}\left(x^{*}\right), \psi_{0}\left(x^{\prime \prime}\right)\right) \leq \lambda\left\|x^{\prime \prime}-x^{\prime}\right\|,
$$

so condition (b) of Lemma 2.1 is satisfied. Hence $\psi_{0}$ has a fixed point $x_{1} \in$ $\mathbb{B}_{r_{0}}\left(x^{*}\right)$.

Proof of Theorem 3.1. Keep $\eta_{0}=x^{*}$ and set $r:=r_{k}=C\left\|x^{*}-x_{k}\right\|$. Proposition 3.2 applied to the map $\psi_{k}$ gives the existence of a fixed point $x_{k+1}$ for $\psi_{k}$, which is an element of $\mathbb{B}_{r_{k}}\left(x^{*}\right)$. This finishes the proof of Theorem 3.1.

Now, we are interested in the sequence $\left(y_{n}\right)$ given by the variant (1.6) of Newton's method. We present a linear convergence result for algorithm (1.6) under the condition (1.7). Before stating the main result, we give some notations. For $\left(y_{k}\right)$ defined in (1.6), define the set-valued mappings $P: X \rightarrow$ $2^{Y}$ and $\Gamma_{k}: X \rightarrow 2^{X}$ by

$$
P(\cdot):=f\left(x^{*}\right)+h \nabla f\left(x^{*}\right)\left(\cdot-x^{*}\right)+G(\cdot), \quad \Gamma_{k}(\cdot):=P^{-1}\left(W_{k}(\cdot)\right),
$$

where $W_{k}$ is defined by

$$
W_{k}(x):=f\left(x^{*}\right)+h \nabla f\left(x^{*}\right)\left(x-x^{*}\right)-f\left(y_{k}\right)-h \nabla f\left(y_{k}\right)\left(x-y_{k}\right) .
$$

We will make the following assumptions in an open convex neighborhood $V$ of $x^{*}$ :

$(\mathcal{H} 0)^{\star}$ There exists $K>0$ such that $\left\|\nabla f\left(y_{0}\right)\right\|<K$.

$(\mathcal{H} 2)^{\star}$ The set-valued map $\left(f\left(x^{*}\right)+h \nabla f\left(x^{*}\right)\left(\cdot-x^{*}\right)+G(\cdot)\right)^{-1}$ is $M^{\prime}-$ pseudo-Lipschitz around $\left(0, x^{*}\right)$ with constants $a^{\prime}$ and $b^{\prime}$ (given by the definition of Aubin continuity) and $M^{\prime}\left((1+|h|) \omega\left(a^{\prime}\right)+\right.$ $|1-h| K)<1$. 
The main result is as follows.

TheOREM 3.3. Let $x^{*}$ be a solution of (1.1). Suppose that assumptions $(\mathcal{H} 0)^{\star},(\mathcal{H} 1)$ and $(\mathcal{H} 2)^{\star}$ are satisfied. For every constant $C^{\prime}$ such that

$$
\frac{M^{\prime}\left(\omega\left(a^{\prime}\right)+|1-h| K\right)}{1-M^{\prime}|h| \omega\left(a^{\prime}\right)}<C^{\prime}<1,
$$

one can find $\gamma>0$ such that for $y_{0} \neq x^{*}$ in $\mathbb{B}_{\gamma}\left(x^{*}\right)$, there exists a sequence $\left(y_{k}\right)$ defined by (1.6) satisfying

$$
\left\|y_{k+1}-x^{*}\right\| \leq C^{\prime}\left\|y_{k}-x^{*}\right\| .
$$

The proof of Theorem 3.3 is based on the following proposition.

Proposition 3.4. Under the assumptions of Theorem 3.3, one can find $\gamma>0$ such that for every starting point $y_{0} \neq x^{*}$ in $\mathbb{B}_{\gamma}\left(x^{*}\right)$, the set-valued map $\Gamma_{0}$ has a fixed point $y_{1}$ in $\mathbb{B}_{\gamma}\left(x^{*}\right)$ satisfying

$$
\left\|y_{1}-x^{*}\right\| \leq C^{\prime}\left\|y_{0}-x^{*}\right\|
$$

where $C^{\prime}$ is as in Theorem 3.3.

Proof. The proposition can be proved in the same way as Proposition 3.2. The constant $\gamma$ is selected such that

$$
\gamma<\min \left\{a^{\prime}, \frac{b^{\prime}}{(1+|h|) \omega\left(a^{\prime}\right)+|1-h| K}\right\} .
$$

Using assumptions $(\mathcal{H} 0)^{\star},(\mathcal{H} 1),(\mathcal{H} 2)^{\star}$ and Lemma 2.2, obtain

$$
\begin{aligned}
\left\|W_{0}\left(x^{*}\right)\right\|= & \left\|f\left(x^{*}\right)-f\left(y_{0}\right)-h \nabla f\left(y_{0}\right)\left(x^{*}-y_{0}\right)\right\| \\
\leq & \left\|f\left(x^{*}\right)-f\left(y_{0}\right)-\nabla f\left(y_{0}\right)\left(x^{*}-y_{0}\right)\right\| \\
& +|1-h|\left\|\nabla f\left(y_{0}\right)\right\|\left\|y_{0}-x^{*}\right\| \\
\leq & \left(\omega\left(\left\|y_{0}-x^{*}\right\|\right)+|1-h|\left\|\nabla f\left(y_{0}\right)\right\|\right)\left\|y_{0}-x^{*}\right\| \\
\leq & \left(\omega\left(a^{\prime}\right)+|1-h| K\right)\left\|y_{0}-x^{*}\right\| .
\end{aligned}
$$

Thus $W_{0}\left(x^{*}\right) \in \mathbb{B}_{b^{\prime}}(0)$. Consequently,

$$
\operatorname{dist}\left(x^{*}, \Gamma_{0}\left(x^{*}\right)\right) \leq M\left(\omega\left(a^{\prime}\right)+|1-h| K\right)\left\|y_{0}-x^{*}\right\| .
$$

As $C^{\prime}\left(1-M^{\prime}|h| \omega\left(a^{\prime}\right)\right)>M^{\prime}\left(\omega\left(a^{\prime}\right)+|1-h| K\right)$, there is $\lambda^{\prime} \in\left[M^{\prime}|h| \omega\left(a^{\prime}\right), 1[\right.$ such that $C^{\prime}\left(1-\lambda^{\prime}\right) \geq M^{\prime}\left(\omega\left(a^{\prime}\right)+|1-h| K\right)$ and

$$
\operatorname{dist}\left(x^{*}, \Gamma_{0}\left(x^{*}\right)\right) \leq C^{\prime}\left(1-\lambda^{\prime}\right)\left\|y_{0}-x^{*}\right\| .
$$

Choosing $r_{0}^{\prime}=C^{\prime}\left\|y_{0}-x^{*}\right\|$, we have $r_{0}^{\prime} \leq \gamma \leq a^{\prime}$. Moreover, for $x \in \mathbb{B}_{\gamma}\left(x^{*}\right)$ 
we obtain

$$
\begin{aligned}
\left\|W_{0}(x)\right\|= & \left\|f\left(x^{*}\right)+h \nabla f\left(x^{*}\right)\left(x-x^{*}\right)-f\left(y_{0}\right)-h \nabla f\left(y_{0}\right)\left(x-y_{0}\right)\right\| \\
\leq & \left\|f\left(x^{*}\right)-f\left(y_{0}\right)-\nabla f\left(y_{0}\right)\left(x^{*}-y_{0}\right)\right\| \\
& +|h|\left\|\nabla f\left(x^{*}\right)-\nabla f\left(y_{0}\right)\right\|\left\|x-x^{*}\right\| \\
& +|1-h|\left\|\nabla f\left(y_{0}\right)\right\|\left\|y_{0}-x^{*}\right\| \\
\leq & \left((1+|h|) \omega\left(a^{\prime}\right)+|1-h| K\right) \gamma .
\end{aligned}
$$

Thus by (3.17) we deduce that $W_{0}(x) \in \mathbb{B}_{b^{\prime}}(0)$ for all $x \in \mathbb{B}_{\gamma}\left(x^{*}\right)$. It follows that for all $x^{\prime}, x^{\prime \prime} \in \mathbb{B}_{r_{0}^{\prime}}\left(x^{*}\right)$,

$$
\begin{aligned}
e\left(\Gamma_{0}\left(x^{\prime}\right) \cap \mathbb{B}_{r_{0}^{\prime}}\left(x^{*}\right), \Gamma_{0}\left(x^{\prime \prime}\right)\right) & \leq M^{\prime}\left\|W_{0}\left(x^{\prime}\right)-W_{0}\left(x^{\prime \prime}\right)\right\| \\
& =M^{\prime}|h|\left\|\left(\nabla f\left(x^{*}\right)-\nabla f\left(y_{0}\right)\right)\left(x^{\prime \prime}-x^{\prime}\right)\right\| \\
& \leq M^{\prime}|h|\left\|\nabla f\left(x^{*}\right)-\nabla f\left(x_{0}\right)\right\|\left\|x^{\prime \prime}-x^{\prime}\right\| \\
& \leq M^{\prime}|h| \omega\left(a^{\prime}\right)\left\|x^{\prime \prime}-x^{\prime}\right\| \leq \lambda^{\prime}\left\|x^{\prime \prime}-x^{\prime}\right\| .
\end{aligned}
$$

The existence of a fixed point $y_{1} \in \mathbb{B}_{r_{0}^{\prime}}\left(x^{*}\right)$ for the map $\Gamma_{0}$ is ensured. This finishes the proof of Proposition 3.4. Theorem 3.3 is deduced by induction.

REMARK 3.5. Theorems 3.1 and 3.3 remain true under the center-Lipschitz condition (1.8).

\section{References}

[1] I. K. Argyros, The Newton-Kantorovich method under mild differentiability conditions and the Pták error estimates, Monatsh. Math. 101 (1990), 175-193.

[2] - On the solution of generalized equations using $m(m \geq 2)$ Fréchet differential operators, Comm. Appl. Nonlinear Anal. 9 (2002), 85-89.

[3] -, A unifying local-semilocal convergence analysis and applications for two-point Newton-like methods in Banach space, J. Math. Anal. Appl. 298 (2004), 374-397.

[4] - Concerning the "terra incognita" between convergence regions of two Newton methods, Nonlinear Anal. 62 (2005), 179-194.

[5] - Relaxing the convergence conditions for Newton-like methods, J. Appl. Math. Comput. 21 (2006), 119-126.

[6] J.-P. Aubin, Lipschitz behavior of solutions to convex minimization problems, Math. Oper. Res. 9 (1984), 87-111.

[7] J.-P. Aubin and H. Frankowska, Set-Valued Analysis, Birkhäuser, Boston, 1990.

[8] A. L. Dontchev, Local convergence of the Newton method for generalized equations, C. R. Acad. Sci. Paris Sér. I 322 (1996), 327-331.

[9] A. L. Dontchev and W. W. Hager, An inverse function theorem for set-valued maps, Proc. Amer. Math. Soc. 121 (1994), 481-489.

[10] A. L. Dontchev, M. Quincampoix and N. Zlateva, Aubin criterion for metric regularity, J. Convex Anal. 13 (2006), 281-297.

[11] M. A. Hernández, The Newton method for operators with Hölder continuous first derivative, J. Optim. Theory Appl. 109 (2001), 631-648.

[12] M. A. Hernández and M. J. Rubio, A modification of Newton's method for nondifferentiable equations, J. Comput. Appl. Math. 164/165 (2004), 323-330. 
[13] S. Hilout, Steffensen-type methods on Banach spaces for solving generalized equations, Adv. Nonlinear Var. Inequal., in press, 2007.

[14] —, A uniparametric Newton-Steffensen-type method for perturbed generalized equations, ibid., in press, 2007.

[15] —, Superlinear convergence of a family of two-step Steffensen-type methods for generalized equations, Int. J. Pure Appl. Math., to appear.

[16] S. Hilout and A. Piétrus, A semilocal convergence of a secant-type method for solving generalized equations, Positivity 10 (2006), 673-700.

[17] C. Jean-Alexis and A. Piétrus, A variant of Newton's method for generalized equations, Rev. Colombiana Mat. 39 (2005), 97-112.

[18] B. S. Mordukhovich, Complete characterization of openness, metric regularity, and Lipschitzian properties of multifunctions, Trans. Amer. Math. Soc. 340 (1993), 1-35.

[19] - Stability theory for parametric generalized equations and variational inequalities via nonsmooth analysis, ibid. 343 (1994), 609-657.

[20] A. Piétrus, Generalized equations under mild differentiability conditions, Rev. R. Acad. Cienc. Madrid 94 (2000), 15-18.

[21] S. M. Robinson, Generalized equations and their solutions, part I: basic theory, Math. Programming Study 10 (1979), 128-141.

[22] —, Generalized equations and their solutions, part II: applications to nonlinear programming, ibid. 19 (1982), 200-221.

[23] R. T. Rockafellar, Lipschitzian properties of multifunctions, Nonlinear Anal. 9 (1984), 867-885.

[24] R. T. Rockafellar and R. J.-B. Wets, Variational Analysis, Grundlehren Math. Wiss. 317, Springer, 1998.

Department of Mathematical Sciences

Cameron University

Lawton, OK 73505, U.S.A.

E-mail: ioannisa@cameron.edu
Department of Applied Mathematics and Computation Faculty of Science and Technics of Béni-Mellal B.P. 523, Béni-Mellal 23000, Morocco E-mail: said_hilout@yahoo.fr 
